\title{
Dynamical taxonomy of the coupled solar radiation pressure and oblateness problem and analytical deorbiting configurations
}

\author{
Ioannis Gkolias ${ }^{1,4} \cdot$ Elisa Maria Alessi ${ }^{2,3}$ D $\cdot$ Camilla Colombo $^{1}$ \\ Received: 9 July 2020 / Revised: 24 October 2020 / Accepted: 3 November 2020 / \\ Published online: 27 November 2020 \\ (c) The Author(s) 2020
}

\begin{abstract}
Recent works demonstrated that the dynamics caused by the planetary oblateness coupled with the solar radiation pressure can be described through a model based on singly averaged equations of motion. The coupled perturbations affect the evolution of the eccentricity, inclination and orientation of the orbit with respect to the Sun-Earth line. Resonant interactions lead to non-trivial orbital evolution that can be exploited in mission design. Moreover, the dynamics in the vicinity of each resonance can be analytically described by a resonant model that provides the location of the central and hyperbolic invariant manifolds which drive the phase space evolution. The classical tools of the dynamical systems theory can be applied to perform a preliminary mission analysis for practical applications. On this basis, in this work we provide a detailed derivation of the resonant dynamics, also in non-singular variables, and discuss its properties, by studying the main bifurcation phenomena associated with each resonance. Last, the analytical model will provide a simple analytical expression to obtain the area-to-mass ratio required for a satellite to deorbit from a given altitude in a feasible timescale.
\end{abstract}

Keywords Solar radiation pressure - Oblateness - Averaged dynamics · Equilibrium points · Bifurcation diagrams · Deorbiting

Elisa Maria Alessi

elisamaria.alessi@cnr.it

Ioannis Gkolias

ioannis.gkolias@polimi.it

Camilla Colombo

camilla.colombo@polimi.it

https://www.compass.polimi.it

1 Department of Aerospace Science and Technology, Politecnico di Milano, Via la Masa 34, 20156 Milan, Italy

2 Istituto di Matematica Applicata e Tecnologie Informatiche "Enrico Magenes", Consiglio Nazionale delle Ricerche, Via Alfonso Corti 12, 20133 Milan, Italy

3 Istituto di Fisica Applicata "Nello Carrara", Consiglio Nazionale delle Ricerche, Via Madonna del Piano 10, 50019 Sesto Fiorentino (FI), Italy

4 Department of Physics, Aristotle University of Thessaloniki, 54124 Thessaloniki, Greece 


\section{Introduction}

The effect of the solar radiation pressure (SRP) on Earth satellites was recognised since the first space flights. The orbital evolution of Vanguard I (Musen et al. 1960) and the Echo balloons (Shapiro and Jones 1960) was found to be significantly influenced by SRP, and singly averaged equations were used to study the motion (Musen 1960). Treated as a perturbation problem, the singly averaged contribution of SRP is integrable and analytical solutions can be obtained (Mignard and Henon 1984; Oyama et al. 2008; Scheeres 2012). Nevertheless, when coupled with the effect of Earth's oblateness $J_{2}$ the system becomes a 2.5 degrees of freedom $(\mathrm{DoF})$. An analytical insight can be recovered when treating locally the semi-secular SRP resonances. Namely, the singly averaged perturbing function can be decomposed in six distinct terms (Kaula 1962), each of them dominating in a particular range of orbital elements. The dynamics arising by combining each of the harmonics with the secular evolution due to $J_{2}$ can be reduced to a 1 DoF resonant model (Krivov and Getino 1997; Lücking et al. 2012; Alessi et al. 2018a).

The derivation of the six different resonant models in the three-dimensional case and their effect on the long-term evolution of resident space objects has been recently discussed in the literature (Alessi et al. 2018a, 2019). In this work, we re-derive the resonant models in the Hamiltonian framework, providing also a non-singular representation of the resonant dynamics. We exploit the information of the analytical model in Alessi et al. (2019) to obtain further insight in the resonant structures. We employ the equations for computing the equilibria for each resonance and compute the number and their stability for each set of the dynamical parameters of the system. This allows us to construct bifurcations diagrams which give the main transitions in the phase space. Particular focus is given to the structure of the phase space about the vicinity of each SRP resonance. The effect of the engineering parameter, the spacecraft area-to-mass ratio, is also discussed.

Using the phase space portraits, useful information for mission design concepts is retrieved via the tools of dynamical system theory. In particular, a rigorous procedure to obtain deorbiting conditions along the resonances is presented. As for the planar case (Lücking et al. 2012), we show how the deorbiting can occur.

The paper is organised in the following way: in Sect. 2 the basic force model is recovered in its singly averaged formulation, in Sect. 3 we discuss the analytical description in the vicinity of SRP resonances, in Sect. 4 we provide a phase space analysis based on the main bifurcations associated with each resonance, in Sect. 5 we demonstrate the use of the models to analytically obtain feasible deorbiting configurations, and in Sect. 6 we present our conclusions.

\section{Model derivation}

Let us assume that a spacecraft moves under the effect of a planet's gravitational monopole, the planetary oblateness and the solar radiation pressure. Moreover, we assume that the sun rays are always perpendicular to the surface of the satellite (cannonball model), that the effect of the planetary albedo is negligible, that the solar flux is constant at $1 \mathrm{au}$, and that the satellite is entirely in sunlight. Under these assumptions, SRP is modelled as a constant force in the direction of the Earth-Sun line, and it can be derived from a potential function. The dynamics of the satellite in a geocentric equatorial inertial frame can be modelled by the Hamiltonian

$$
\mathcal{H}=\mathcal{H}_{\text {kep }}+\mathcal{H}_{J_{2}}+\mathcal{H}_{\text {SRP }}
$$


The Keplerian part $\mathcal{H}_{\text {kep }}$ reads

$$
\mathcal{H}_{\text {kep }}=\frac{v^{2}}{2}-\frac{\mu}{r},
$$

where $\mu$ is the gravitational parameter of the Earth, and $r, v$ are the geocentric distance and velocity of the satellite.

The Earth's oblateness effect is modelled as

$$
\mathcal{H}_{J_{2}}=\frac{\mathcal{C}_{J_{2}}\left(3 \sin ^{2} \phi-1\right)}{2 r^{3}},
$$

where $\mathcal{C}_{J_{2}}=\mu R_{\oplus}^{2} J_{2}$ with $J_{2}$ the oblateness parameter and $\phi$ the geographic latitude of the satellite. The sine of the latitude is expressed in terms of the orbital elements of the satellite via

$$
\sin \phi=\frac{z}{r}
$$

and

$$
z=(0,0,1) R_{3}(-\Omega) R_{1}(-i) R_{3}(-\lambda)(r, 0,0)^{\mathrm{T}},
$$

where $i$ is the inclination, $\Omega$ the Right Ascension of the Ascending Node (RAAN), $\lambda=\omega+f$ the argument of latitude, $\omega$ the argument of perigee and $f$ the true anomaly of the satellite. The rotation matrices $R_{1}(u), R_{3}(u)$ are

$$
R_{1}(u)=\left(\begin{array}{ccc}
1 & 0 & 0 \\
0 & \cos u & \sin u \\
0 & -\sin u & \cos u
\end{array}\right), \quad R_{3}(u)=\left(\begin{array}{ccc}
\cos u & \sin u & 0 \\
-\sin u & \cos u & 0 \\
0 & 0 & 1
\end{array}\right)
$$

The solar radiation pressure contribution is given by

$$
\mathcal{H}_{\text {SRP }}=\mathcal{C}_{\text {SRP }} X
$$

where $\mathcal{C}_{\mathrm{SRP}}=P_{\odot} c_{R} \frac{A}{m}, P_{\odot}$ being the SRP constant at $1 A U, c_{R}$ the reflectivity coefficient and $\frac{A}{m}$ the area-to-mass ratio of the satellite. $X$ is the coordinate of the satellite in an Earthcentred system with the X-axis pointed towards the Sun (see Fig. 1): in terms of the orbital elements of the satellite it reads

$$
X=(1,0,0) R_{3}\left(\lambda_{\odot}\right) R_{1}(\varepsilon) R_{3}(-\Omega) R_{1}(-i) R_{3}(-\theta)(r, 0,0)^{\mathrm{T}},
$$

with $\lambda_{\odot}$ the ecliptic longitude of the Sun and $\varepsilon$ the obliquity of the ecliptic.

Both $\mathcal{H}_{J_{2}}$ and $\mathcal{H}_{\text {SRP }}$ are considerable smaller than $\mathcal{H}_{\text {kep }}$ and can be treated as perturbations of the two-body problem

$$
\mathcal{H}=\mathcal{H}_{\text {kep }}+\mathcal{H}_{\text {per }}
$$

where $\mathcal{H}_{\text {per }}=\mathcal{H}_{J_{2}}+\mathcal{H}_{\text {SRP. }}$ Employing a Hori-Deprit approach (Hori 1966; Deprit 1969), the homological equation up to the first order of the normalisation process reads:

$$
n \frac{\partial W_{1}}{\partial M}=\mathcal{H}_{\text {per }}-\overline{\mathcal{H}}_{\text {per }},
$$

where $n$ is the mean motion of the satellite, $W_{1}$ is the generating function, and

$$
\overline{\mathcal{H}}_{\text {per }}=\frac{1}{2 \pi} \int_{0}^{2 \pi} \mathcal{H}_{\text {per }} \mathrm{d} M,
$$


Fig. 1 Schematic description of an equatorial inertial and an ecliptic rotating reference frames. The solar radiation pressure is modelled as a constant force in the direction of the Sun

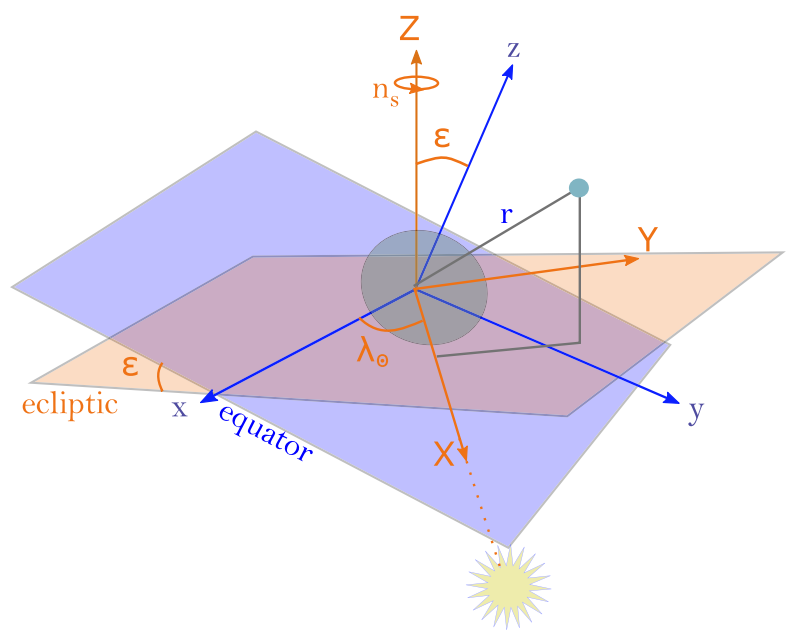

is the first-order normalized Hamiltonian. The integrals in Eq. (2) can be carried out in closed form for both the $J_{2}$ and SRP contributions. In the case of $J_{2}$ we use the differential relationship $\mathrm{d} M=\frac{r^{2}}{a \sqrt{1-e^{2}}} \mathrm{~d} f$ along with $r=a \frac{1-e^{2}}{1+e \cos f}$ to obtain the classical result

$$
\overline{\mathcal{H}}_{J_{2}}=\frac{\mathcal{C}_{J_{2}}\left(3 c_{i}^{2}-1\right)}{4 a^{3}\left(1-e^{2}\right)^{3 / 2}},
$$

where $c_{i}=\cos i$. For SRP we express the integral with respect to the eccentric anomaly $E$ using the relations $r \sin f=a \sqrt{1-e^{2}} \sin E, r \cos f=a(\cos E-e), r=a(1-e \cos E)$ and $\mathrm{d} M=\frac{r}{a} \mathrm{~d} E$.

The averaged model is then (Krivov and Getino 1997; Lücking et al. 2012; Alessi et al. 2018a)

$$
\overline{\mathcal{H}}_{\mathrm{SRP}}=-\frac{3}{2} a e \mathcal{C}_{\mathrm{SRP}} \sum_{j=1}^{6} \mathcal{T}_{j} \cos \psi_{j},
$$

with

\begin{tabular}{|c|c|c|}
\hline$j$ & $\mathcal{T}_{j}$ & $\cos \psi_{j}$ \\
\hline \hline 1 & $\frac{1}{4}\left(c_{\varepsilon}+1\right)\left(c_{i}+1\right)$ & $\cos \left(\omega+\Omega-\lambda_{\odot}\right)$ \\
2 & $-\frac{1}{4}\left(c_{\varepsilon}+1\right)\left(c_{i}-1\right)$ & $\cos \left(-\omega+\Omega-\lambda_{\odot}\right)$ \\
3 & $\frac{1}{2} s_{i} s_{\varepsilon}$ & $\cos (\omega-\lambda \odot)$ \\
4 & $-\frac{1}{2} s_{i} s_{\varepsilon}$ & $\cos \left(\omega+\lambda_{\odot}\right)$ \\
5 & $-\frac{1}{4}\left(c_{\varepsilon}-1\right)\left(c_{i}+1\right)$ & $\cos \left(\omega+\Omega+\lambda_{\odot}\right)$ \\
6 & $\frac{1}{4}\left(c_{\varepsilon}-1\right)\left(c_{i}-1\right)$ & $\cos \left(-\omega+\Omega+\lambda_{\odot}\right)$ \\
\hline
\end{tabular}

where $c_{i}, s_{i}, c_{\varepsilon}, s_{\varepsilon}$ are the cosine and sine of the inclination $i$ and the obliquity of the ecliptic $\varepsilon$, respectively. The model consists of 6 distinct harmonics describing the semi-secular evolution of the system, all of them containing the ecliptic longitude of the Sun $\lambda \odot$. A second averaging over the Sun's mean motion results in a null Hamiltonian, that is, the SRP perturbation does not give rise to secular effects. Moreover, we should mention that Hamiltonian Eq. (4) is integrable, and an analytical solution is obtained using an ecliptic rotating frame (Mignard and Henon 1984). 
The singly averaged model of the coupled Earth's oblateness and solar radiation pressure effects reads

$$
\overline{\mathcal{H}}=\overline{\mathcal{H}}_{J_{2}}+\overline{\mathcal{H}}_{\text {SRP }}
$$

The Hamiltonian can be expressed in terms of the canonical Delaunay elements $(L, G, H, l, g, h)$ such that

$$
\begin{array}{ll}
L=\sqrt{\mu a}, & l=M, \\
G=L \sqrt{1-e^{2}}, & g=\omega, \\
H=G \cos i, & h=\Omega,
\end{array}
$$

to obtain

$$
\overline{\mathcal{H}}=\frac{\mathcal{C}_{J_{2}} \mu^{3}\left(G^{2}-3 H^{2}\right)}{4 G^{5} L^{3}}-\frac{3 \mathcal{C}_{\mathrm{SRP}} \sqrt{1-\frac{G^{2}}{L^{2}}} L^{2}}{2 \mu} \sum_{j=1}^{6} \mathcal{T}_{j} \cos \psi_{j},
$$

where for the coefficients $\mathcal{T}_{j}$ we use the relationships $c_{i}=H / G, s_{i}=\sqrt{1-\frac{H^{2}}{G^{2}}}$.

Due to the averaging process, Eq. (7) does not depend on the mean anomaly $M=l$ and thus the Delaunay action $L$, as well as the semi-major axis, is constant. The system has two degrees-of-freedom (DoF) and an explicit time dependence through $\lambda_{\odot}(t)=\lambda_{\odot, 0}+n_{s} t$, where $n_{s}$ is the frequency corresponding to Earth's orbital period of 1 year. Considering an extended phase space, a dummy action $I_{s}$ with frequency $n_{s}$ is added to the Hamiltonian

$$
\overline{\mathcal{H}}=\frac{\mathcal{C}_{J_{2}} \mu^{3}\left(G^{2}-3 H^{2}\right)}{4 G^{5} L^{3}}-\frac{3 \mathcal{C}_{\mathrm{SRP}} \sqrt{1-\frac{G^{2}}{L^{2}}} L^{2}}{2 \mu} \sum_{j=1}^{6} \mathcal{T}_{j} \cos \psi_{j}+n_{s} I_{s},
$$

which yields a three DoF autonomous system.

\subsection{Semi-secular solar gravitational resonances}

As a side note, we recall that the second-order singly averaged Hamiltonian perturbative term corresponding to the solar gravitational attraction, say $\overline{\mathcal{H}}_{3 b S}$, includes the same resonant arguments reported in (5). In particular,

$$
\begin{aligned}
& \overline{\mathcal{H}}_{3 b S}^{1}=-\frac{15 a^{2} e^{2} \mu_{S}\left(c_{\varepsilon}+1\right)^{2}\left(c_{i}+1\right)^{2} \cos \left(2 \psi_{1}\right)}{128 a_{S}^{3}}, \\
& \overline{\mathcal{H}}_{3 b S}^{2}=-\frac{15 a^{2} e^{2} \mu_{S}\left(c_{\varepsilon}+1\right)^{2}\left(c_{i}-1\right)^{2} \cos \left(2 \psi_{2}\right)}{128 a_{S}^{3}}, \\
& \overline{\mathcal{H}}_{3 b S}^{3}=\frac{15 a^{2} e^{2} \mu_{S}\left(c_{\varepsilon}^{2}-2 s_{\varepsilon}^{2}-1\right) s_{i}^{2} \cos \left(2 \psi_{3}\right)}{64 a_{S}^{3}}, \\
& \overline{\mathcal{H}}_{3 b S}^{4}=\frac{15 a^{2} e^{2} \mu_{S}\left(c_{\varepsilon}^{2}-2 s_{\varepsilon}^{2}-1\right) s_{i}^{2} \cos \left(2 \psi_{4}\right)}{64 a_{S}^{3}}, \\
& \overline{\mathcal{H}}_{3 b S}^{5}=-\frac{15 a^{2} e^{2} \mu_{S}\left(c_{\varepsilon}-1\right)^{2}\left(c_{i}+1\right)^{2} \cos \left(2 \psi_{5}\right)}{128 a_{S}^{3}}, \\
& \overline{\mathcal{H}}_{3 b S}^{6}=-\frac{15 a^{2} e^{2} \mu_{S}\left(c_{\varepsilon}-1\right)^{2}\left(c_{i}-1\right)^{2} \cos \left(2 \psi_{6}\right)}{128 a_{S}^{3}},
\end{aligned}
$$


where $\mu_{S}$ is the solar gravitational parameter and $a_{S}$ the geocentric semi-major axis of the Sun.

In the following, we will assume that the range of $(a, e)$ and the value of area-to-mass ratio are such that the perturbation effects corresponding to $\overline{\mathcal{H}}_{3 b S}$ are negligible. This is, for $j$ given,

$$
\left|\frac{\overline{\mathcal{H}}_{\text {SRP }}^{j}}{\overline{\mathcal{H}}_{3 b S}^{j}}\right| \gg 1
$$

For the specific application presented at the end of paper (Sect.5), we will verify this hypothesis.

\section{Resonances}

We are now interested in studying the resonance phenomena occurring in the coupled system. A resonance is occurring when the following commensurability holds:

$$
n_{1} \dot{g}+n_{2} \dot{h}+n_{3} \dot{\lambda}_{\odot} \approx 0
$$

where $\dot{g}=\partial \overline{\mathcal{H}} / \partial G, \dot{h}=\partial \overline{\mathcal{H}} / \partial H$ and $\dot{\lambda}_{\odot}=\partial \overline{\mathcal{H}} / \partial I_{s}$. The integer coefficients $n_{1}, n_{2}, n_{3}$ can take the values of the 6 combinations associated with the harmonics appearing in Eq. (5). As a first approximation, the angular frequencies can be computed by taking into account only the $H_{J_{2}}$ part of the Hamiltonian, namely,

$$
\begin{aligned}
& \dot{g}_{J_{2}}=\frac{\partial \overline{\mathcal{H}}}{\partial G}=-\frac{3\left(G^{2}-5 H^{2}\right) \mathcal{C}_{J_{2}} \mu^{3}}{4 G^{6} L^{3}}=-\frac{3}{2} \frac{J_{2} R_{\oplus}^{2} n}{a^{2}\left(1-e^{2}\right)^{2}} \cos i, \\
& \dot{h}_{J_{2}}=\frac{\partial \overline{\mathcal{H}}}{\partial H}=-\frac{3 H \mathcal{C}_{J_{2}} \mu^{3}}{2 G^{5} L^{3}}=\frac{3}{4} \frac{J_{2} R_{\oplus}^{2} n}{a^{2}\left(1-e^{2}\right)^{2}}\left(5 \cos ^{2} i-1\right), \\
& \dot{\lambda}_{\odot}=\frac{\partial \overline{\mathcal{H}}}{\partial I_{S}}=n_{s} .
\end{aligned}
$$

Substituting Eq. (10) in Eq. (9), we can derive an approximation of the loci in action space of the centre of each resonance (Cook 1962; Alessi et al. 2018a). In the vicinity of each resonance, the associate harmonic is slow and is dominating the dynamics. Assuming an isolated resonance approximation, the system can be described in the vicinity of the particular resonance by the Hamiltonian

$$
\mathcal{H}_{\psi_{j}}=\frac{\mathcal{C}_{J_{2}} \mu^{3}\left(G^{2}-3 H^{2}\right)}{4 G^{5} L^{3}}-\frac{3 \mathcal{C}_{\mathrm{SRP}} \sqrt{1-\frac{G^{2}}{L^{2}}} L^{2}}{2 \mu} \mathcal{T}_{j} \cos \psi_{j}+n_{s} I_{s} .
$$

For each resonance with argument $\psi_{j}$ (with $j=1 \ldots 6$ ) we introduce a resonant set of variables $(\Psi, \psi)$ via a unimodular transformation of the Delaunay elements

$$
\begin{array}{rlrl}
\Psi & =\frac{G}{n_{1}}, & \psi & =n_{1} g+n_{2} h+n_{3} \lambda_{\odot}, \\
\Pi=-n_{2} G+n_{1} H, & \pi=\frac{h}{n_{1}}, \\
K=I_{s}-\frac{n_{3} G}{n_{1}} . & \kappa & =\lambda_{\odot} .
\end{array}
$$


which brings the Hamiltonian to a 1-DoF form:

$$
H_{\psi_{j}}=H_{\psi_{j}, J_{2}}(\Psi ; L, \Pi)+H_{\psi_{j}, S R P}(\Psi, \psi ; L, \Pi)+n_{s}\left(n_{3} \Psi+K\right),
$$

where the part associated with $J_{2}$ is

$$
\mathcal{H}_{\psi_{j}, J_{2}}(\Psi ; L, \Pi)=\frac{C_{J_{2}} \mu^{3}\left(n_{1}^{4} \Psi^{2}-3\left(\Pi+n_{1} n_{2} \Psi\right)^{2}\right)}{n_{1}^{7} 4 L^{3} \Psi^{5}},
$$

and the one due to SRP is

$$
\mathcal{H}_{\psi_{j}, S R P}(\Psi, \psi ; L, \Pi)=-\frac{3 C_{\mathrm{SRP}}}{2 \mu} L^{2} \sqrt{1-\frac{n_{1}^{2} \Psi^{2}}{L^{2}}} \mathcal{T}_{j} \cos \psi,
$$

while the coefficients $\mathcal{T}_{j}$ are expressed in terms of the new variables using the equations $c_{i}=\frac{n_{1} n_{2} \Psi+\Pi}{n_{2}^{2} \Psi}$ and $s_{i}=\sqrt{1-c_{i}^{2}}$.

Due to the resonant transformation both $\pi$ and $\kappa$ are ignorable; therefore, $\Pi$ and $K$ are constants and the term $n_{s} K$ can be dropped from the Hamiltonian. The action variable $\Pi$ is a resonant integral of the system; its value is dictated from the initial conditions and remains constant during the orbital evolution. Expressed in orbital elements

$$
\Pi=\sqrt{\mu a} \sqrt{1-e^{2}}\left(-n_{2}+n_{1} \cos i\right)
$$

represents coupled oscillations in the eccentricity and inclination of the orbit, in a similar fashion to the Lidov-Kozai constant in the third-body gravitational perturbations (Lidov 1962; Kozai 1962). Note that $\Pi$ corresponds to $\Lambda$ in Alessi et al. (2019). The formulation provided here is equivalent to the one given in the past paper, but $n_{1}$ and $n_{2}$ are exchanged.

For a satellite close to a resonance with argument $\psi_{j}$ with mean initial elements

$$
\left(a_{0}, e_{0}, i_{0}, \omega_{0}, \Omega_{0}, \lambda_{\odot, 0}\right),
$$

the orbit evolution in the $(\Psi, \psi)$ plane, or equivalently in the $(e, \psi)$ plane if we substitute $\Psi=\sqrt{\mu a\left(1-e^{2}\right)} / n_{1}$, is given from the contour line of the implicit equation:

$$
H_{\psi_{j}}(\Psi, \psi ; L, \Pi)=H_{\psi_{j}}\left(\Psi\left(a_{0}, e_{0}\right), \psi\left(\omega_{0}, \Omega_{0}, \lambda_{\odot, 0}\right) ; L, \Pi\right),
$$

with $L=L\left(a_{0}\right), \Pi=\Pi\left(a_{0}, e_{0}, i_{0}\right)$. Notice that $\Pi$ depends on the resonance $j$ through $n_{1}$ and $n_{2}$.

The equations of the motion related to the resonant models $H_{\psi_{j}}$ are

$$
\begin{aligned}
\frac{\mathrm{d} \psi}{\mathrm{d} t} & =\frac{\partial H_{\psi_{j}}}{\partial \Psi}, \\
\frac{\mathrm{d} \Psi}{\mathrm{d} t} & =-\frac{\partial H_{\psi_{j}}}{\partial \psi},
\end{aligned}
$$

and the associated equilibria are given from the equations

$$
\begin{aligned}
\frac{\mathrm{d} \psi}{\mathrm{d} t} & =0 \\
\frac{\mathrm{d} \Psi}{\mathrm{d} t} & =0
\end{aligned}
$$

Those stationary solutions of the resonant model represent periodic orbits of the full equations of motion. Their stability is determined from the eigenvalues of the Hessian of the Hamiltonian $\mathcal{H}_{\psi_{j}}$ computed at each equilibrium point. 
In many applications the equations of motion with respect to the eccentricity are of interest, and thus, by applying the chain rule for the derivatives and using the relations $\Psi=G / n_{1}$ and $\mathrm{d} e / \mathrm{d} G=-\frac{\sqrt{1-e^{2}}}{e \sqrt{\mu a}}$ we can derive the semi-canonical form:

$$
\begin{aligned}
\frac{\mathrm{d} e}{\mathrm{~d} t} & =n_{1} \frac{\sqrt{1-e^{2}}}{e \sqrt{\mu a}} \frac{\partial H_{\psi_{j}}}{\partial \psi}=n_{1} \mathcal{C}_{\mathrm{SRP}} \frac{\sqrt{1-e^{2}}}{n a} \mathcal{T}_{j} \sin \psi \\
\frac{\mathrm{d} \psi}{\mathrm{d} t} & =-n_{1} \frac{\sqrt{1-e^{2}}}{e \sqrt{\mu a}} \frac{\partial H_{\psi_{j}}}{\partial e}=n_{1} \dot{g}_{\left(J_{2}, \psi_{j}\right)}+n_{2} \dot{h}_{\left(J_{2}, \psi_{j}\right)}+n_{3} n_{s} .
\end{aligned}
$$

and

$$
\begin{aligned}
& \dot{g}_{\left(J_{2}, \psi_{j}\right)}=\dot{g}_{J_{2}}+\dot{g}_{\psi_{j}}, \\
& \dot{h}_{\left(J_{2}, \psi_{j}\right)}=\dot{h}_{J_{2}}+\dot{h}_{\psi_{j}},
\end{aligned}
$$

where $\dot{g}_{J_{2}}, \dot{h}_{J_{2}}$ are given in Eq. (10) and

$$
\begin{aligned}
& \dot{h}_{\psi_{j}}=\frac{\partial H_{\psi_{j}, S R P}}{\partial H}=C_{\mathrm{SRP}} \frac{e}{n a \sqrt{1-e^{2}} \sin i} \frac{\partial \mathcal{T}_{j}}{\partial i} \cos \psi, \\
& \dot{g}_{\psi_{j}}=\frac{\partial H_{\psi_{j}, S R P}}{\partial G}=C_{\mathrm{SRP}} \frac{\sqrt{1-e^{2}}}{n a e} \mathcal{T}_{j} \cos \psi-\dot{h}_{\psi_{j}} \cos i,
\end{aligned}
$$

that is the model developed in Alessi et al. (2019).

In all the above formulation the angles $g, h$ are not well defined when the eccentricity and/or the inclination is zero. To alleviate this burden we use the modified Delaunay variables in Eq. (11)

$$
\begin{array}{ll}
\Lambda=L, & \lambda=l+g+h, \\
P=L-G, & p=-g-h, \\
Q=G-H, & q=-h,
\end{array}
$$

while a unimodular transformation allows to derive a new set of resonant variables $(\Sigma, \sigma)$, similar to the ones introduced in Breiter (2001) for the case of lunisolar resonances, namely,

$$
\begin{aligned}
\Sigma & =\frac{P}{k_{1}}, & \sigma & =k_{1} p+k_{2} q+k_{3} \lambda_{\odot}, \\
\Phi & =-k_{2} P+k_{1} Q, & \phi & =\frac{q}{k_{1}}, \\
\Gamma & =I_{s}-\frac{k_{3} P}{k_{1}}, & \gamma & =\lambda_{\odot} .
\end{aligned}
$$

where $k_{1}, k_{2}, k_{3}$ are integers appearing in combinations associated with each harmonic in Eq. (4)

\begin{tabular}{|c|c|c|}
\hline$j$ & $\cos \psi_{j}$ & $\left(k_{1}, k_{2}, k_{3}\right)$ \\
\hline \hline 1 & $\cos \left(-p-\lambda_{\odot}\right)$ & $(\mp 1,0, \mp 1)$ \\
2 & $\cos \left(-p+2 q-\lambda_{\odot}\right)$ & $(\mp 1, \pm 2, \mp 1)$ \\
3 & $\cos \left(-p+q-\lambda_{\odot}\right)$ & $(\mp 1, \pm 1, \mp 1)$ \\
4 & $\cos \left(-p+q+\lambda_{\odot}\right)$ & $(\mp 1, \pm 1, \pm 1)$ \\
5 & $\cos \left(-p+\lambda_{\odot}\right)$ & $(\mp 1,0, \pm 1)$ \\
6 & $\left.\cos \left(-p+2 q+\lambda_{\odot}\right)\right)$ & $(\mp 1, \pm 2, \pm 1)$ \\
\hline
\end{tabular}


After a Taylor expansion in the resonant action $\Sigma$ and dropping the constant terms, the resonant Hamiltonian up to a truncation order $N$ takes the form

$$
H_{\psi_{j}}^{(N)}=n_{s} k_{3} \Sigma+C_{J_{2}} \sum_{n=1}^{N} c_{n} \Sigma^{n}+C_{\mathrm{SRP}} \sum_{n=1}^{N} d_{n} \Sigma^{n-1 / 2} \cos \sigma,
$$

where $c_{n}, d_{n}$ are constant coefficients depending on the dynamical parameters $(L, \Phi)$, the particular resonance $\left(k_{1}, k_{2}\right)$ and the physical parameters $(\varepsilon, \mu)$. We notice that up to third order $(N=3)$ the resonant model is similar to the extended fundamental model introduced in Breiter (2001) and analysed in Breiter (2003). In practice, a truncation order of $N \geq 4$ is required to retrieve the qualitative features of the phase space, while higher orders are used for more accurate computations. Actually, the phase-space exploration can be done with the non-truncated resonant Hamiltonian and we retain the complete dynamical portrait of the resonance, a fact that highlights the power of the closed-form averaging process.

Finally, it is common to express the resonant Hamiltonian in terms of Poincaré variables

$$
X=\sqrt{2 \Sigma} \cos \sigma, \quad Y=\sqrt{2 \Sigma} \sin \sigma,
$$

with $x=\frac{X}{\sqrt{L}} \sim e \cos \sigma$ and $y=\frac{X}{\sqrt{L}} \sim e \sin \sigma$ providing a polar representation of the resonant domain, while the truncated Hamiltonian in Eq. (23) takes a polynomial form in $x, y$ coordinates.

\section{Bifurcation analysis}

From the equations written above it is possible to compute the equilibrium points associated with the dynamical system together with the corresponding stability. The isolated resonance system has two constants of motion (apart from $\mathcal{H}$ ): the semi-major is constant due to the averaging and the second integral $\Pi$ from Eq. (15). The values of $\Pi$ can be labelled based on the inclination of the corresponding circular orbit $\Pi \equiv \Pi_{\text {circ }}=n_{1} \cos i_{\text {circ }} \sqrt{\mu a}-n_{2}$. Given a set of values of the dynamical parameters $\left(a, i_{\text {circ }}\right)$ and the engineering parameter $A / m$, a phase space is uniquely identified. In this phase space the number of equilibrium points and their stability can be defined. By varying the parameters and tracking the structural changes in the system, a bifurcation diagram can be computed. Elliptic and saddle points appear and disappear based on the classical bifurcation theory for 1-DOF systems.

For the two harmonics that dominate in the low Earth orbit region, namely, $\psi_{1}$ and $\psi_{2}$ (Alessi et al. 2018b), in Fig. 2 and in Fig. 4 the possible phase space structures are depicted in the range of semi-major axis $a \in$ [7000:9400] km. The black curves define the boundaries of the regions characterized by a different number of equilibrium points and corresponding stability. In red, the curves that would be obtained by considering only the oblateness effect for the rate of precession of $\omega$ and $\Omega$ are shown, that is, only Eq. (10) instead of Eq. (18). For the resonant harmonic $\psi_{1}$ in Fig. 2 we present two bifurcation diagrams for $A / m=$ $1 \mathrm{~m}^{2} / \mathrm{kg}$ (left) and $A / m=5 \mathrm{~m}^{2} / \mathrm{kg}$ (right). There are 5 distinct dynamical regions labelled with Latin numbers, defined in Table 1, the corresponding phase spaces being shown in Fig. 3. As just mentioned, the red line corresponds to the resonance location based on the $J_{2}$ rates. We observe that for the low $A / m=1 \mathrm{~m}^{2} / \mathrm{kg}$ value, this line almost coincides with the transition from region I to region III and from I to II. From Table 1 it is easy to see that the following bifurcations take place:

$\psi=0$ : 

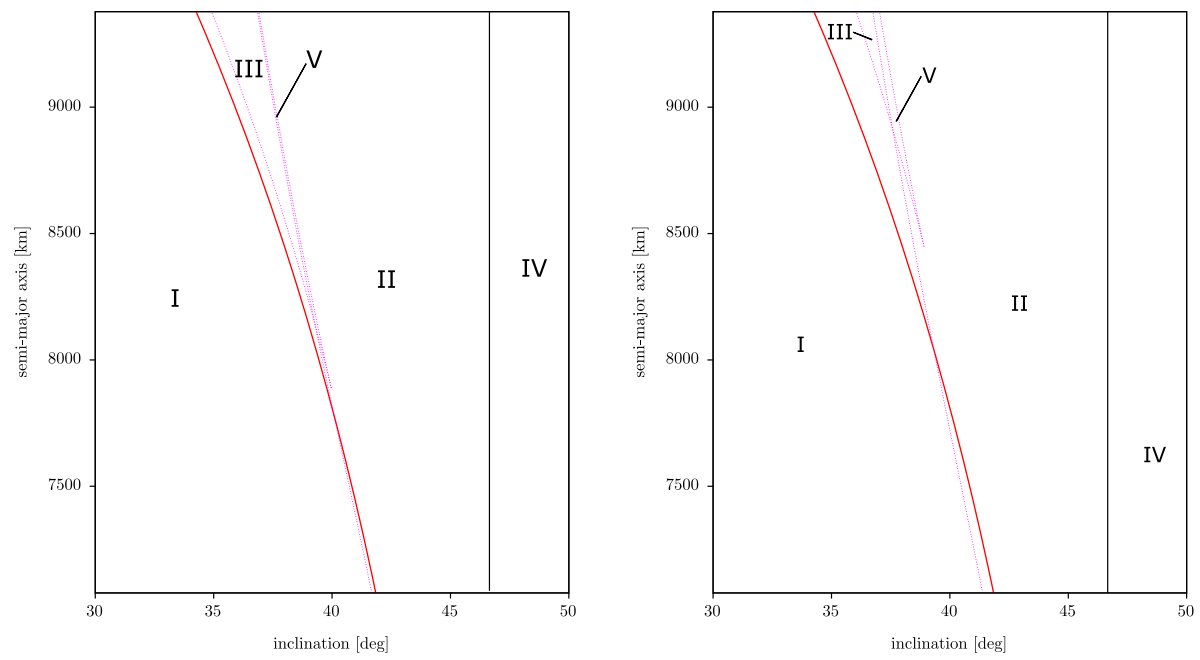

Fig. 2 The bifurcation diagram for the resonance with argument $\psi_{1}$. The total number of equilibria is presented in the dynamical parameter space $\left(a, i_{\text {circ }}\right)$. Left: $A / m=1 \mathrm{~m}^{2} / \mathrm{kg}$; right: $A / m=5 \mathrm{~m}^{2} / \mathrm{kg}$. The red line corresponds to the resonant condition computed from solely the $J_{2}$ rate. Inclination stands for inclination of the circular orbit. For the type of equilibria in each region see Table 1

Table 1 Number of equilibria and their stability (s: stable, u: unstable) for the resonance with argument $\psi_{1}$, corresponding to the five regions of Fig. 2

\begin{tabular}{llll}
\hline Region & Total & $\psi_{1}=0$ & $\psi_{1}=\pi$ \\
\hline I & 3 & $1 \mathrm{~s}$ & $1 \mathrm{~s}$ and $1 \mathrm{u}$ \\
II & 1 & $1 \mathrm{~s}$ & - \\
III & 5 & $2 \mathrm{~s}$ and $1 \mathrm{u}$ & $1 \mathrm{~s}$ and $1 \mathrm{u}$ \\
IV & 3 & $1 \mathrm{~s}$ and $1 \mathrm{u}$ & $1 \mathrm{~s}$ \\
V & 3 & $2 \mathrm{~s}$ and $1 \mathrm{u}$ & - \\
\hline
\end{tabular}

The corresponding phase space is depicted in Fig. 3

- transition from region I to region III: saddle-centre;

- transition from region V to region II: saddle-centre;

$\psi=\pi$ :

- transition from region III to region V: saddle-centre;

- transition from region I to region II: saddle-centre.

The effect of an increased $A / m$ on the bifurcation diagram is shown in the right panel of Fig. 2. In this case, the exact position of the bifurcation is no longer predicted from the $J_{2}$ only rates as significant contributions are added to the $\dot{\omega}$ and $\dot{\Omega}$ rates due to SRP. The number of regions and their structure remain the same; however, the boarders between the different regions considerably alter. Specifically, for a fixed $i_{\text {circ }}$ the bifurcation for region I to regions II and III seems to happen at higher altitudes. This is in agreement with previous findings on the planar case $\left(i_{\text {circ }}=0\right)$ of $\psi_{1}$ (Lücking et al. 2012).

A similar analysis is now performed for the resonance with argument $\psi_{2}$. Assuming the same range semi-major axis as in $\psi_{1}$ case, we expect this harmonic to dominate in a range of inclinations from $i \in$ [76:84]. The bifurcation diagram is shown in Fig. 4, and the equilibria with their associated stability are given in Table 2. 
Fig. 3 Resonant phase space portraits for $\psi_{1}$, in $e-\psi$ and $x-y$ variables, according to the regions defined in Table 1 (top to bottom)
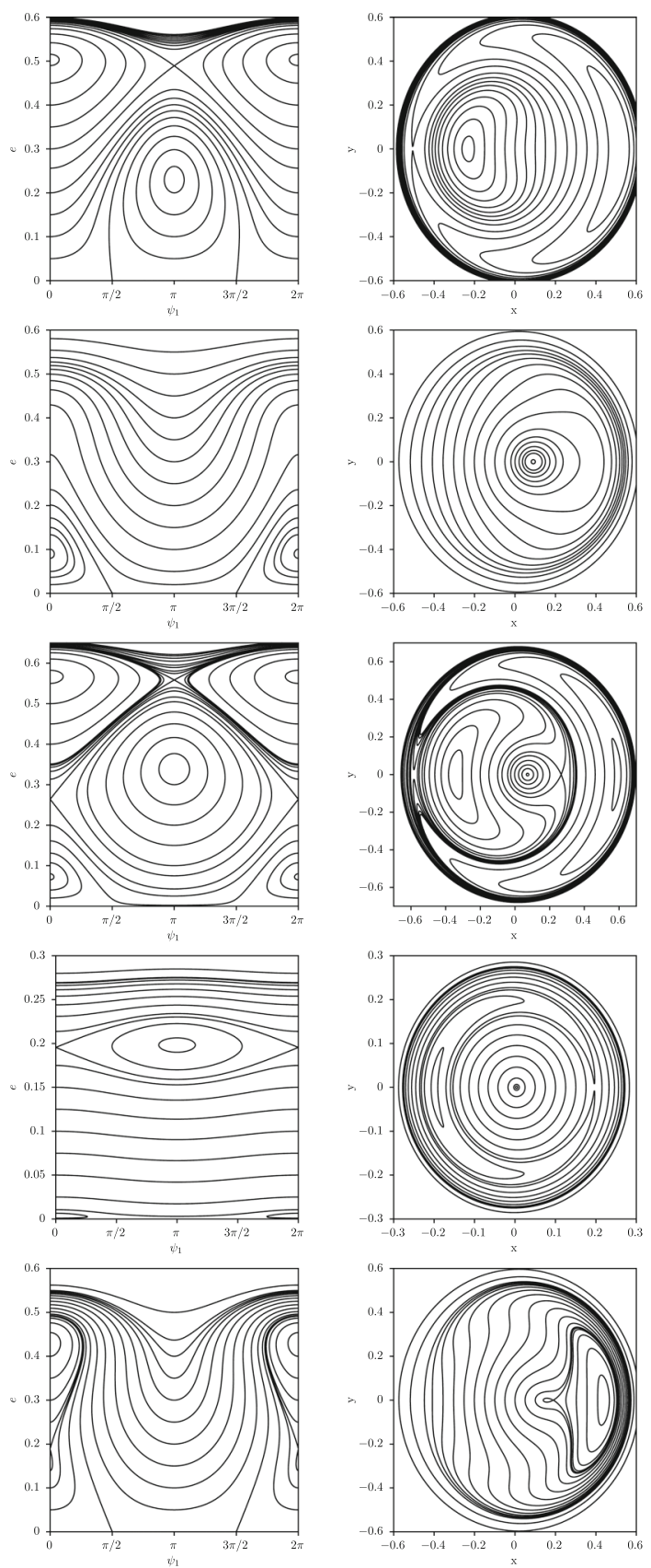
Fig. 4 The bifurcation diagram $\psi_{2}$ assuming $A / m=1 \mathrm{~m}^{2} / \mathrm{kg}$. The total number of equilibria is presented in the dynamical parameter space $\left(a, i_{\text {circ }}\right)$. The red line corresponds to the resonant condition computed from solely the $J_{2}$ rate. Inclination stands for inclination of the circular orbit. For the type of equilibria in each region see Table 2 for the resonance with argument

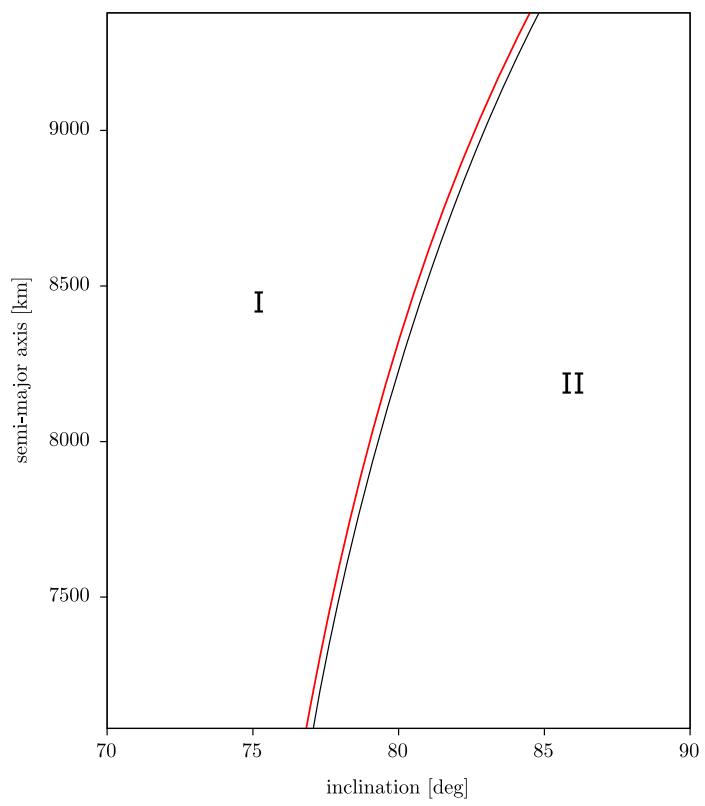

Table 2 Number of equilibria and their stability (s: stable, u: unstable) for the resonance with argument $\psi_{2}$, corresponding to the two regions of Fig. 4

\begin{tabular}{llll}
\hline Region & Total & $\psi_{2}=0$ & $\psi_{2}=\pi$ \\
\hline I & 1 & - & $1 \mathrm{~s}$ \\
II & 3 & $1 \mathrm{~s}$ and $1 \mathrm{u}$ & $1 \mathrm{~s}$ \\
\hline
\end{tabular}

The situation in this case appears to be more straightforward. There are two distinct regions: in region I there exists only 1 stable equilibrium at $\psi_{2}=\pi$, while in region II two more equilibria, 1 stable and unstable, appear at $\psi_{2}=0$ after another saddle-centre bifurcation. The position of the bifurcation for $A / m=1 \mathrm{~m}^{2} / \mathrm{kg}$ is approximately estimated from the $J_{2}$ resonant condition (red curve in Fig. 4).

Figure 5 extends the analysis by displaying the bifurcation diagrams for resonances $\psi_{1}$, $\psi_{3}$ and $\psi_{4}$, assuming $A / m=1 \mathrm{~m}^{2} / \mathrm{kg}$ and a range of semi-major axis $a \in$ [7000:20000]. As also found in Alessi et al. (2019), the maximum number of equilibrium points is 5 also for $\psi_{4}$, and it is interesting to note that for $\psi_{1}$ it can occur another type of bifurcation apart from the saddle-centre: the transcritical one beyond $a \sim 17000 \mathrm{~km}$ and $i_{\text {circ }} \in\left[130^{\circ}: 150^{\circ}\right]$. For the same resonant argument $\psi_{1}$, we can detect the saddle connection bifurcation, as described in Breiter (2003). An example is shown in Fig. 6: in red it is highlighted the separatrix that connects two saddle points.

The bifurcation diagram for $\psi_{5}$ and $\psi_{2}$ is symmetrical with respect to $i_{\text {circ }}=90^{\circ}$, the same holds for $\psi_{6}$ and $\psi_{1}$.

\section{Deorbiting configuration}

The phase space analysis can be exploited to compute the initial conditions, in terms of orbital elements and area-to-mass ratio, that can lead to an atmospheric reentry. Since the semi-major 

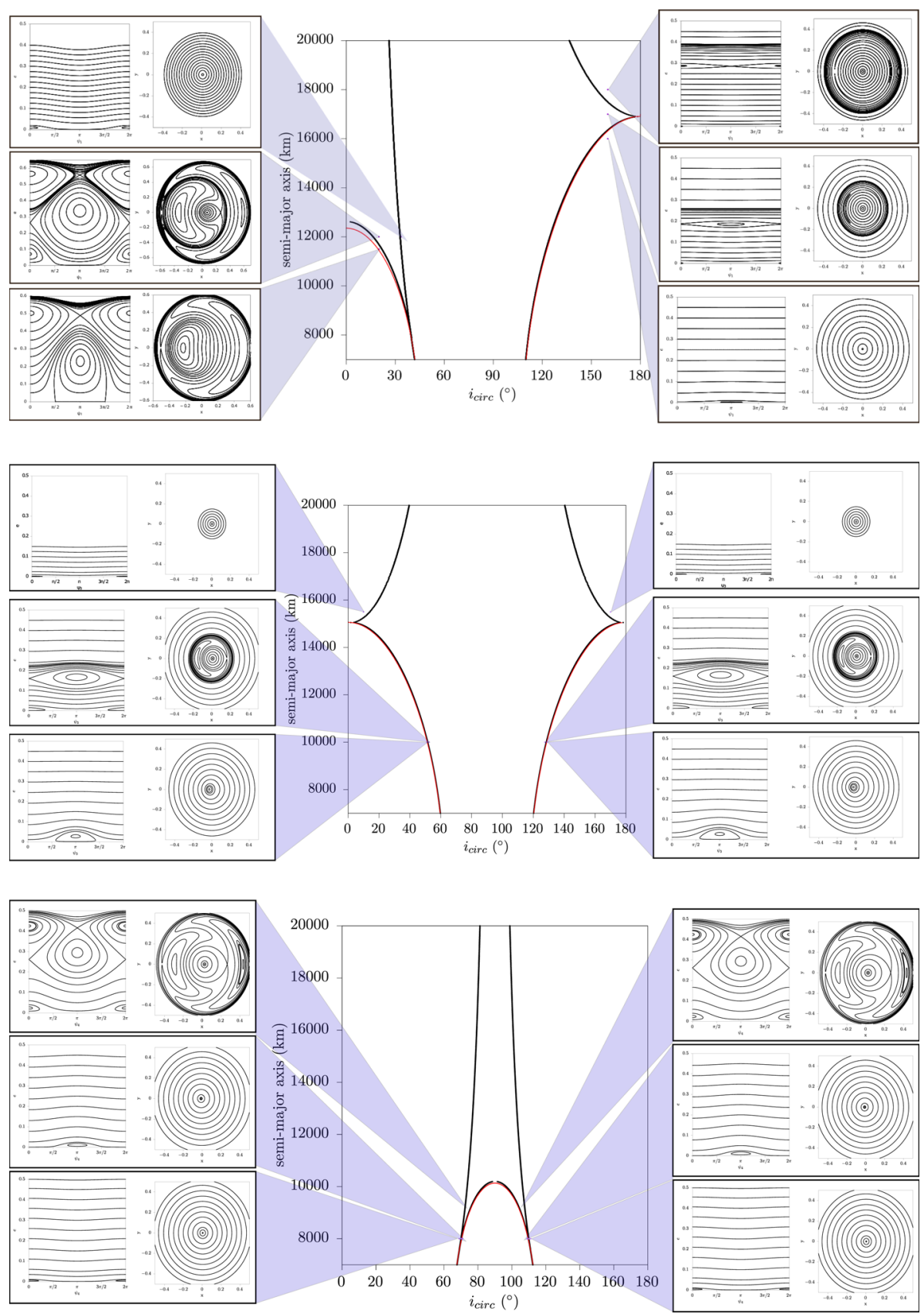

Fig. 5 The bifurcation diagram for the resonances with arguments $\psi_{1}$ (top), $\psi_{3}$ (middle) and $\psi_{4}$ (bottom). The red curves correspond to the bifurcation that is obtained considering only the oblateness effect in $\dot{\omega}$ and $\dot{\Omega}$ 

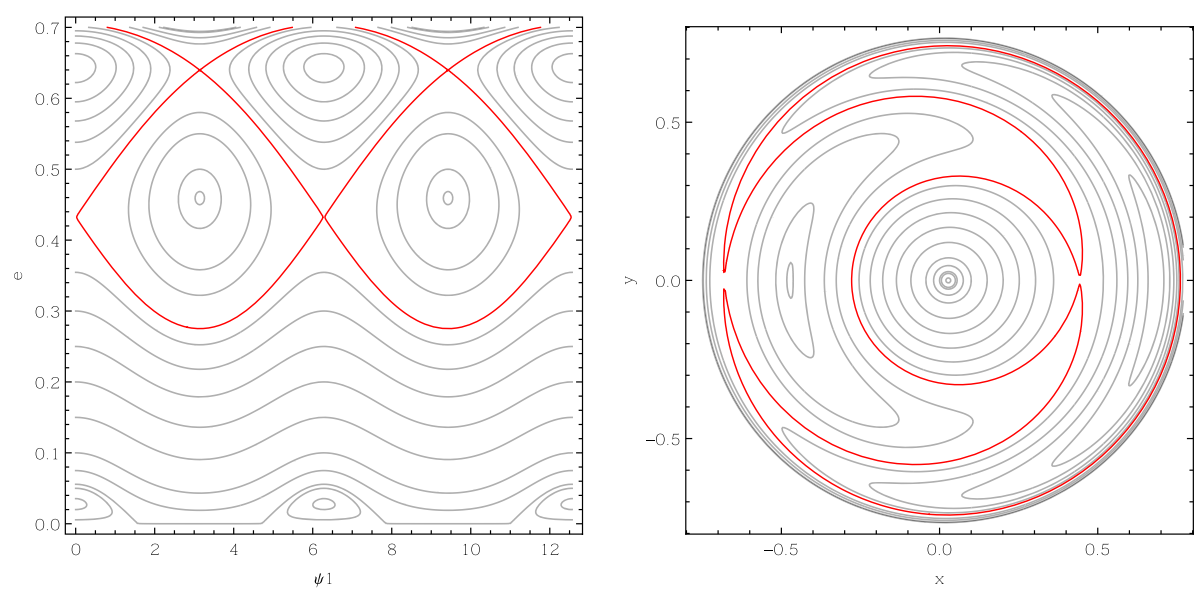

Fig. 6 Resonant phase space portrait for $\psi_{1}$, in $e-\psi$ and $x-y$ variables, in the case of a saddle connection (in red). On the left we extended the $\psi_{1}$-range to highlight the connection between the two saddle points

axis does not change under the dynamics considered, a natural deorbiting can take place only if the eccentricity increases as much as to attain the critical value $e_{\mathrm{cr}}=1-r_{\oplus} / a$.

We consider the case where this condition occurs at either $\psi=0$ or $\psi=\pi$, meaning that the area-to-mass ratio required is the minimum. Moreover, mindful of the phase space behaviour depicted in the previous section (see Fig. 3), the steepest eccentricity growth from a circular orbit takes place starting from $\psi=\pi / 2 \vee 3 \pi / 2$ and following the stable direction associated with a hyperbolic equilibrium point or a libration curve associated with an elliptic equilibrium point. Based on this and following the idea presented in Lücking et al. (2013) for the planar case, the initial conditions that can enable a natural deorbiting from a circular orbit in the spatial case can be obtained by solving the following equation

$$
\mathcal{H}_{\psi_{j}}(\Psi(e=0), \psi ; L, \Pi)-\mathcal{H}_{\psi_{j}}\left(\Psi\left(e=e_{\mathrm{cr}}\right), \psi ; L, \Pi\right)=0, \quad \psi=0 \vee \pi,
$$

as a function of $C_{\mathrm{SRP}}$ (or, equivalently, $A / m$ ). In particular,

$$
\begin{aligned}
C_{\mathrm{SRP}}= \pm & \frac{\mu}{6 L^{10} n_{1}^{2} \mathcal{T}_{j} \eta_{\mathrm{cr}}^{5} e_{\mathrm{cr}}}\left\{4 L^{9} n_{1} n_{3} n_{s}\left(\eta_{\mathrm{cr}}-1\right) \eta_{\mathrm{cr}}^{5}\right. \\
& -\mathcal{C}_{J_{2}} L^{2}\left(n_{1}^{2}-3 n_{2}^{2}\right) \eta_{\mathrm{cr}}^{2}\left(\eta_{\mathrm{cr}}^{3}-1\right) \mu^{3} \\
& \left.+6 \mathcal{C}_{J_{2}} L n_{2} \eta_{\mathrm{cr}}\left(-1+\eta_{\mathrm{cr}}^{4}\right) \mu^{3} \Pi+3 \mathcal{C}_{J_{2}}\left(\eta_{\mathrm{cr}}^{5}-1\right) \mu^{3} \Pi^{2}\right\}
\end{aligned}
$$

where $\eta_{\mathrm{cr}}=\sqrt{1-e_{\mathrm{cr}}^{2}}$, and the coefficients $T_{j}$ are computed using $c_{i}=\frac{L n_{2} \eta_{\mathrm{cr}}+\Pi}{L n_{1} \eta_{\mathrm{cr}}}$ and $s_{i}=\sqrt{1-c_{i}^{2}}$. The \pm sign depends on whether the critical eccentricity is attained at $\psi=0$ or $\psi=\pi$, respectively. Note that only the positive solutions are admissible, because it represents a physical area-to-mass ratio. Moreover, it should be noticed that the solution does not provide any information on the time required to cover the invariant curve up to $e=e_{\mathrm{cr}}$.

In the above expression, the only unknown is $\Pi$ that can be set considering that the deorbiting starts from $e_{0}=0$ and $\psi_{0}=\pi / 2 \vee 3 \pi / 2$, following a resonant curve. Given the value of semi-major axis $a$, the value of the inclination can be found by computing the resonant condition $\dot{\psi}=0$ at that configuration. Following Alessi et al. (2019), the resonant condition 

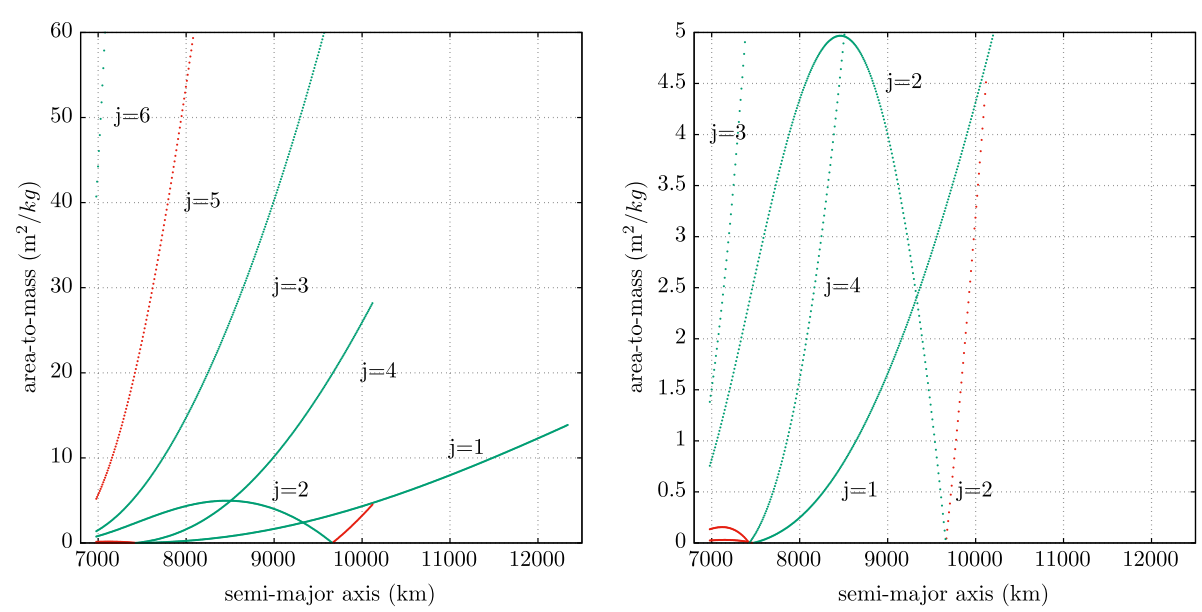

Fig. 7 Minimum area-to-mass ratio required to obtain a natural deorbiting, according to Eq. (26). Red: the deorbiting occurs at $\psi=0$; green: at $\psi=\pi$. Right: a closer view at feasible values of $A / m$

$\dot{\psi}=0$ at $\psi=\pi / 2 \vee 3 \pi / 2$ does not depend on the solar radiation pressure, but only on the oblateness effect. In particular, $i_{0}$ can be obtained by solving the quadratic equation in $\cos i_{0}$ (Alessi et al. 2019)

$$
c_{1} \cos ^{2} i_{0}+c_{2} \cos i_{0}+c_{3}=0,
$$

with

$$
c_{1}=\frac{15 n_{2} J_{2} r_{\oplus}^{2} n}{4 a^{2}\left(1-e^{2}\right)^{2}} ; \quad c_{2}=-\frac{3 n_{1} J_{2} r_{\oplus}^{2} n}{2 a^{2}\left(1-e^{2}\right)^{2}} ; \quad c_{3}=n_{3} n_{S}-\frac{3 n_{2} J_{2} r_{\oplus}^{2} n}{4 a^{2}\left(1-e^{2}\right)^{2}}
$$

\subsection{Results}

We have applied the procedure just described to the range $a \in[6978: 15000] \mathrm{km}$, considering a discretization of $\Delta a=10 \mathrm{~km}$. The lower limit for the range in $a$ reflects the fact that below about $600 \mathrm{~km}$ of altitude a circular orbit decays naturally in 25 years due to the effect of the atmospheric drag.

In Fig. 7, we show the area-to-mass ratio computed by means of Eq. (26), as a function of initial semi-major axis for the six resonant arguments, for prograde orbits. The colour reports if the deorbiting occurs at $\psi=0$ (red) or $\psi=\pi$ (green). It should be noticed that, as already detected numerically (Alessi et al. 2018b; Schaus et al. 2019; Rossi et al. 2020), the resonant terms $j=5,6$ are the least effective ones, requiring very high values of $A / m$. Moreover, the conditions depicted always correspond to a libration curve.

The area-to-mass range $A / m \in[1: 5] \mathrm{m}^{2} / \mathrm{kg}$ is characteristic of solar sails that are feasible with the current technology developments (Colombo 2018). These are devices we can envisage to embed a spacecraft to deorbit them passively at the end of life. Higher values can represent clouds of fragments of space debris and can be used, for instance, to see whether specific corridors are actually followed by fragments reentering the Earth after a collision. The option of setting specific observational campaigns can be explored in the future on the basis of the analysis presented here. 

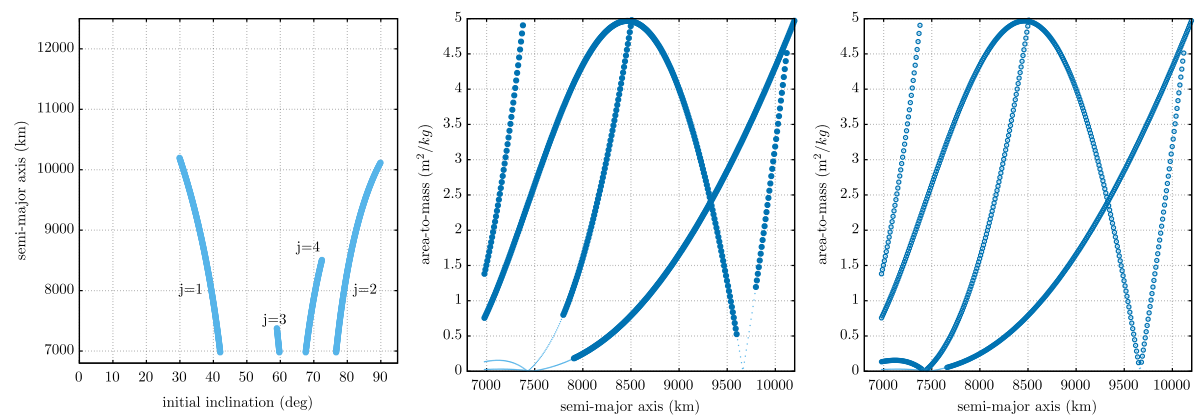

Fig. 8 Left: semi-major axis and inclination for the circular orbits corresponding to the minimum area-tomass depicted in Fig. 7 on the right. Middle: the blue solid thicker points correspond to the conditions that can deorbit in less than 50 years. Right: the blue empty thicker points correspond to the conditions such that the approximation of neglecting the solar semi-secular terms is valid

In Fig. 8, we show, on the left, the value of initial inclination and semi-major axis corresponding to the conditions depicted in Fig. 7 on the right. In the middle, we show with a thicker solid point the configurations that actually attain $e_{\mathrm{cr}}$ in less than 50 years. Such limit can be considered conservative, because in the numerical propagation only the Earth's oblateness and the SRP are taken into account. The atmospheric drag, that will speed up the reentry for altitudes below $700 \mathrm{~km}$ (or higher, depending on $A / \mathrm{m}$ ), is neglected. The cases where a 50-year reentry is not achieved are associated with two different behaviours:

- either the dynamics is too slow and this happens, in particular, after the cusps that can be noticed in the figure;

- or there exist two curves, not connected, associated with the same value of $\mathcal{H}$, for a same integral of motion $\Lambda$. This happens, in particular, for $j=1$ and $j=4$ before for values of semi-major axis lower than $a=7500 \mathrm{~km}$.

In same Fig. 8, on the right, we display with a thicker empty point the configurations such that the hypothesis of neglecting the semi-secular solar gravitational perturbations is valid (recall Sect. 2.1). We consider that the approximation is valid if the ratio between the two effects is larger than 1000. It can be noticed that this is true in all the relevant cases shown in the middle panel.

\section{Conclusions}

In this work, we have extended the analytical development of the equations of motion associated with the coupled oblateness-solar radiation pressure effect. In particular, the Hamiltonian formulation, only partially explained in Alessi et al. (2018a, 2019), has been here accurately presented. On this basis, the whole phase space in Earth orbit up to $a=20000 \mathrm{~km}$ has been described by means of a dynamical taxonomy that shows where the main bifurcations occur, in particular the saddle-centre, the transcritical and the saddle connection ones. Finally, the analytical value of the area-to-mass ratio required to de-orbit has been provided, for the first time in the three-dimensional case, and the corresponding applications for a feasible disposal strategy by means of a solar sail have been presented.

The model presented is a first approximation of how the solar radiation pressure effect coupled with the planetary oblateness can act on a satellite. The model neglects more complex 
issues, like shadows or short-term effects, but previous numerical works showed that the solutions shown persist when considering a full model that includes the mentioned issues. This aspect was faced in particular in Alessi et al. (2018b) and Schaus et al. (2019).

The coupling with the solar gravitational perturbation that can occur at higher altitudes will be considered in the future.

Acknowledgements This article is part of the COMPASS project, that has received funding from the European Research Council (ERC) under the European Union's Horizon 2020 research and innovation programme (Grant agreement No. 679086). The authors would like to acknowledge the previous studies cited in this paper performed in the framework of the European Commission Horizon 2020 ReDSHIFT project (Grant agreement No. 687500) and collaborations therein. Elisa Maria Alessi would like to acknowledge the support of CNRIFAC and CNR-IMATI for the completion of this work. The authors would like to thank the anonymous reviewers for their useful suggestions.

\section{Compliance with ethical standards}

Conflict of interest On behalf of all authors, the corresponding author states that there is no conflict of interest.

Open Access This article is licensed under a Creative Commons Attribution 4.0 International License, which permits use, sharing, adaptation, distribution and reproduction in any medium or format, as long as you give appropriate credit to the original author(s) and the source, provide a link to the Creative Commons licence, and indicate if changes were made. The images or other third party material in this article are included in the article's Creative Commons licence, unless indicated otherwise in a credit line to the material. If material is not included in the article's Creative Commons licence and your intended use is not permitted by statutory regulation or exceeds the permitted use, you will need to obtain permission directly from the copyright holder. To view a copy of this licence, visit http://creativecommons.org/licenses/by/4.0/.

\section{References}

Alessi, E.M., Schettino, G., Rossi, A.: Solar radiation pressure resonances in low earth orbits. Mon. Not. R. Astron. Soc. 473, 2407-2414 (2018a). https://doi.org/10.1093/mnras/stx2507

Alessi, E.M., Schettino, G., Rossi, A., Valsecchi, G.B.: Natural highways for end-of-life solutions in the LEO region. Celest. Mech. Dyn. Astron. 130, 34 (2018b). https://doi.org/10.1007/s10569-018-9822-z

Alessi, E.M., Colombo, C., Rossi, A.: Phase space description of the dynamics due to the coupled effect of the planetary oblateness and the solar radiation pressure perturbations. Celest. Mech. Dyn. Astron. 131, 43 (2019). https://doi.org/10.1007/s10569-019-9919-z

Breiter, S.: Lunisolar resonances revisited. Celest. Mech. Dyn. Astron. 81, 81-91 (2001)

Breiter, S.: Extended fundamental model of resonance. Celest. Mech. Dyn. Astron. 85(3), 209-218 (2003)

Colombo, C., et al.: Effects of passive deorbiting through drag and solar sails and electrodynamic tethers on the space debris environments. In: 69th International Astronautical Congress. Bremen, Germany (2018). Paper A6.2.8

Cook, G.E.: Luni-solar perturbations of the orbit of an Earth satellite. Geophys. J. 6, 271-291 (1962). https:// doi.org/10.1111/j.1365-246X.1962.tb00351.x

Deprit, A.: Canonical transformations depending on a small parameter. Celest. Mech. 1(1), 12-30 (1969). https://doi.org/10.1007/BF01230629

Hori, G.: Theory of general perturbation with unspecified canonical variable. Publ. Astron. Soc. Jpn. 18, 287 (1966)

Kaula, W.M.: Development of the lunar and solar disturbing functions for a close satellite. Astron. J. 67(5), 300-303 (1962). https://doi.org/10.1086/108729

Kozai, Y.: Secular perturbations of asteroids with high inclination and eccentricity. Astron. J. 67, 591 (1962). https://doi.org/10.1086/108790

Krivov, V.A., Getino, J.: Orbital evolution of high-altitude balloon satellites. Astron. Astrophys. 318, 308-314 (1997) 
Lidov, M.L.: The evolution of orbits of artificial satellites of planets under the action of gravitational perturbations of external bodies. Planet. Space Sci. 9, 719-759 (1962). https://doi.org/10.1016/00320633(62)90129-0

Lücking, C., Colombo, C., McInnes, C.R.: A passive satellite deorbiting strategy for medium earth orbit using solar radiation pressure and the $j_{2}$ effect. Acta Astronaut. 77, 197-206 (2012). https://doi.org/10.1016/ j.actaastro.2012.03.026

Lücking, C., Colombo, C., McInnes, C.R.: Solar radiation pressure-augmented deorbiting: passive end-of-life disposal from high-altitude orbits. J. Spacecr. Rockets 50(6), 1256-1267 (2013). https://doi.org/10.2514/ 1.A32478

Mignard, F., Henon, M.: About an unsuspected integrable problem. Celest. Mech. 33, 239-250 (1984). https:// doi.org/10.1007/BF01230506

Musen, P.: The influence of the solar radiation pressure on the motion of an artificial satellite. J. Geophys. Res. 65(5), 1391-1396 (1960). https://doi.org/10.1029/JZ065i005p01391

Musen, P., Bryant, R., Bailie, A.: Perturbations in perigee height of Vanguard I. Science 131(3404), 935-9366 (1960). https://doi.org/10.1126/science.131.3404.935

Oyama, T., Yamakawa, H., Omura, Y.: Orbital dynamics of solar sails for geomagnetic tail exploration. J. Guid. Control Dyn. 45(2), 316-323 (2008). https://doi.org/10.2514/1.31274

Rossi, A., Alessi, E.M., Schettino, G., Schaus, V., Valsecchi, G.B.: How an aware usage of the long-term dynamics can improve the long-term situation in the LEO region. Acta Astronaut. 174, 159-165 (2020). https://doi.org/10.1016/j.actaastro.2020.05.005

Schaus, V., Alessi, E.M., Schettino, G., Rossi, A., Stoll, E.: On the practical exploitation of perturbative effects in low Earth orbit for space debris mitigation. Adv. Space Res. 63, 1979-1991 (2019). https://doi.org/ 10.1016/j.asr.2019.01.020

Scheeres, D.J.: Orbit mechanics about asteroids and comets. J. Guid. Control Dyn. 35(3), 987-997 (2012). https://doi.org/10.2514/1.57247

Shapiro, I.I., Jones, H.M.: Perturbations of the orbit of the echo balloon. Science 132(3438), 1484-1486 (1960). https://doi.org/10.1126/science.132.3438.1484

Publisher's Note Springer Nature remains neutral with regard to jurisdictional claims in published maps and institutional affiliations. 